\title{
ADMINISTRAÇÃO DE MEDICAMENTOS EM UM SERVIÇO DE EMERGÊNCIA: AÇÕES REALIZADAS E DESAFIOS PARA PRÁTICAS SEGURAS
}

Thais dos Santos Pinheiro ${ }^{1}$ Érica Toledo de Mendonça ${ }^{1}$ Andréia Guerra Siman ${ }^{1}$ Camilo Amaro de Carvalho ${ }^{1}$ Fernando Pacheco Zanelli ${ }^{1}$ Marilane de Oliveira Fani Amaro ${ }^{1}$

\author{
https://orcid.org/0000-0002-9566-973X \\ https://orcid.org/0000-0002-3014-1504 \\ http://orcid.org/0000-0001-7990-9273 \\ https://orcid.org/0000-0001-9539-8653 \\ https://orcid.org/0000-0002-1215-4934 \\ http://orcid.org/0000-0002-9495-0861
}

Objetivo: analisar as ações realizadas e os desafios da equipe de enfermagem para a administração segura de medicamentos no serviço de emergência de um hospital de ensino. Método: Pesquisa qualitativa realizada com 17 profissionais da equipe de enfermagem atuantes no serviço de emergência. A coleta de dados foi realizada através de entrevista com roteiro semiestruturado e os dados foram submetidos à Análise de Conteúdo. Resultados: a primeira categoria revelou as atitudes dos entrevistados frente à administração segura dos medicamentos, tendo a maioria citado ações que contemplam os 9 certos da administração, mas de forma incompleta. A segunda categoria apresentou as dificuldades e desafios que a equipe enfrenta no cotidiano para administração segura dos medicamentos, como elevado número de pacientes atendidos e desconhecimento dos novos medicamentos. Conclusão: depreende-se que a equipe de enfermagem entrevistada atende parcialmente às normas de administração segura dos medicamentos, visto que atua em convergência com algumas ações recomendadas; no entanto, a falta de padronização para a administração dos medicamentos, o alto fluxo de pacientes, a sobrecarga de trabalho e o surgimento de novos medicamentos podem favorecer a ocorrência de eventos adversos. Faz-se necessário investimento em novas tecnologias e educação permanente da equipe no ambiente de trabalho.

Descritores: Enfermagem; Segurança do paciente; Erros de medicação.

\section{ADMINISTRATION OF MEDICINES IN AN EMERGENCY SERVICE: ACTIONS UNDERTAKEN AND CHALLENGES FOR SAFE PRACTICES}

Objective: to analyze the actions taken and the challenges of the nursing team for the safe administration of medications in the emergency department of a teaching hospital. Method: Qualitative research conducted with 17 professionals from the nursing team working in the emergency service. Data collection was carried out through an interview with a semi-structured script and the data were submitted to Content Analysis. Results: the first category revealed the attitudes of the interviewees towards the safe administration of medications, with the majority citing actions that contemplate the 9 right ones from the administration, but in an incomplete way. The second category presented the difficulties and challenges that the team faces in the daily routine for safe administration of medications, such as the high number of patients seen and the lack of knowledge about new medications. Conclusion: it appears that the interviewed nursing team partially complies with the rules for safe administration of medications, since it acts in convergence with some recommended actions; however, the lack of standardization for the administration of medications, the high flow of patients, the work overload and the emergence of new medications can favor the occurrence of adverse events. Investment in new technologies and permanent education of the team in the work environment is necessary.

Descriptors: Nursing; Patient safety; Medication errors

\section{ADMINISTRACIÓN DE DROGAS EN UN SERVICIO DE EMERGENCIA: ACCIONES TOMADAS Y DESAFÍOS PARA PRÁCTICAS SEGURAS}

Objetivo: analizar las acciones tomadas y los desafíos del equipo de enfermería para la administración segura de medicamentos en el departamento de emergencias de un hospital universitario. Método: Investigación cualitativa realizada con 17 profesionales del equipo de enfermería que trabajan en el servicio de emergencia. La recolección de datos se realizó a través de una entrevista con un guión semiestructurado y los datos se enviaron a Content Analysis Resultados: la primera categoría reveló las actitudes de los entrevistados hacia la administración segura de medicamentos, con la mayoría citando acciones que contemplan las 9 correctas de la administración, pero de manera incompleta. La segunda categoría presentó las dificultades y desafios que enfrenta el equipo en la rutina diaria para la administración segura de medicamentos, como la gran cantidad de pacientes atendidos y la falta de conocimiento sobre nuevos medicamentos. Conclusión: parece que el equipo de enfermería entrevistado cumple parcialmente con las reglas para la administración segura de medicamentos, ya que actúa en convergencia con algunas acciones recomendadas; sin embargo, la falta de estandarización para la administración de medicamentos, el alto flujo de pacientes, la sobrecarga de trabajo y la aparición de nuevos medicamentos pueden favorecer la aparición de eventos adversos. Es necesaria la inversión en nuevas tecnologías y la educación permanente del equipo en el entorno laboral.

Descriptores: Enfermería; Seguridad del paciente; Errores de medicación.

'Universidade Federal de Viçosa, MG, Brasil.

Autor correspondente: Marilane de Oliveira Fani Amaro - Email: marilaneamaro@yahoo.com.br

Recebido: 13/02/2020 - Aceito: 19/05/2020 


\section{INTRODUÇÃO}

A qualidade dos serviços de saúde está intimamente relacionada à segurança do paciente, tema que tem sido discutido mundialmente nos últimos anos Em 2004, a Organização Mundial da Saúde (OMS) criou a Aliança Mundial para Segurança do Paciente com o objetivo de promover e desenvolver políticas e práticas referentes ao assunto em seus paises-membros ${ }^{(1)}$.

No Brasil, a temática tomou grande magnitude após o lançamento do Programa Nacional de Segurança do Paciente (PNSP), através da Portaria $n^{\circ} 529 / 2013^{(2)}$, do Ministério da Saúde (MS) e da Resolução da Diretoria Colegiada (RDC) da Agência Nacional de Vigilância Sanitária (ANVISA) 36/2013(3) , cujas diretrizes culminaram na criação do Núcleo de Segurança do Paciente (NSP), que corrobora com o propósito de redução de danos, instituindo ações para melhoria na qualidade da assistência prestada em todos os níveis de atenção à saúde ${ }^{(2-3)}$. A segurança do paciente é definida pela ANVISA como redução dos riscos de danos desnecessários, denominado evento adverso, a um mínimo aceitável ${ }^{(3)}$.

Nesse sentido, o erro de medicação constitui uma séria ameaça à saúde pública, é um desafio aos profissionais envolvidos, e pode ser definido como um evento evitável induzido pelo uso inapropriado do medicamento e com grande potencial para prejudicar a saúde do paciente ${ }^{(4-5)}$.

O processo de administrar medicamentos caracteriza-se por uma atividade dinâmica e complexa, que inclui a produção, a prescrição, a dispensação e a administração, que se inter-relacionam e podem influenciar positivamente na segurança ao paciente, podendo ocorrer em quaisquer desses processos ${ }^{(4)}$

Desta forma, padroniza-se a realização das diversas etapas de modo a resultar em um desfecho favorável, conhecidos como os "9 certos da administração" sendo eles: paciente certo, medicamento certo, via certa, hora certa, dose certa, registro certo, ação certa, forma certa e resposta certa. Os nove certos não garantem que os erros de administração não ocorrerão, mas segui-los pode prevenir significativamente parte desses eventos, resultando em segurança e qualidade da assistência prestada ao paciente ${ }^{(6)}$

Pesquisas apontam outras ações de segurança na redução do erro, como a identificação de medicações de alto risco; utilização de soluções tecnológicas, como prontuário eletrônico; criação de manuais explicativos e mudanças no processo de trabalho. Além disso, antes de administrar o medicamento pode-se utilizar o método de checar os itens corretos por dois enfermeiros, o "check-double" $(4,6,7)$.
A partir dessas premissas, destacam-se as unidades de atendimento de urgência e emergência, como locais caracterizados pela superlotação e alta rotatividade de pacientes, que associados ao estresse, escassez de profissionais e falhas na comunicação da equipe, possuem grande vulnerabilidade aos eventos adversos ${ }^{(4,8)}$. Dentre os profissionais de saúde, a equipe de enfermagem está mais suscetivel a cometer erros, seja pelos procedimentos invasivos que realiza ou pelo tempo de permanência junto ao paciente, e dessa forma assume papel fundamental para preveni-los ${ }^{(9)}$.

Nesse contexto, diante da relevância da correta administração de medicamentos no serviço de emergência, surgiu a seguinte pergunta norteadora: quais ações a equipe de enfermagem tem realizado para alcançar a administração segura de medicamentos em um serviço de emergência? Desta maneira, o presente estudo tem como objetivo analisar as ações realizadas e os desafios da equipe de enfermagem para a administração segura de medicamentos no serviço de emergência de um hospital de ensino.

\section{METODOLOGIA}

\section{Tipo de estudo}

Trata-se de uma pesquisa exploratória, descritiva com abordagem qualitativa. A definição pela linha de pesquisa qualitativa ocorreu devido a sua característica de trabalhar com valores, crenças, representações, hábitos, atitudes e opiniões, e por ser um tipo de investigação interpretativo-formativa, que busca a compreensão do tema pesquisado, favorecendo o processo de descobrimento, por meio de análise, síntese de ideias e conceitos, com envolvimento de aspectos emocionais e contextuais ${ }^{(10)}$.

\section{Local de estudo}

O cenário de estudo foi um serviço de emergência, constituído de 12 leitos e 2 consultórios para realização de pequenos procedimentos. A emergência é de uma instituição hospitalar filantrópica, de médio porte, vinculada como hospital de ensino, localizada no interior de Minas Gerais, Brasil. A instituição pertencia ao ProHosp (Programa de Fortalecimento e Melhoria da Qualidade dos Hospitais do Sistema Único de Saúde de Minas Gerais), o que exigia a criação da comissão de gerenciamento de risco. Portanto, para a definição da instituição como cenário do presente estudo, considerou-se o fato de ser um hospital que possuía uma comissão de gerenciamento de riscos e que desenvolvia ações voltadas para a qualidade e segurança do paciente. A Sistematização da Assistência de enfermagem estava sendo implementada no setor. 


\section{Participantes do estudo}

Como critérios de inclusão dos participantes elegeram-se: ser enfermeiro ou técnico de enfermagem do serviço de emergência, independentemente do tempo de exercício da função. Enfermeiros ou técnicos de enfermagem afastados do cargo por qualquer motivo foram excluidos da pesquisa. No período da coleta de dados, havia um técnico de enfermagem no período de férias. Portanto, os participantes da pesquisa foram 12 técnicos de enfermagem, 4 enfermeiros assistenciais e 1 enfermeiro coordenador.

\section{Coleta de dados}

Os dados foram coletados no período de novembro de 2016 a março de 2017, a partir de observação não participante com registro em diário de campo e entrevista com roteiro semiestruturado elaborado pelos autores do estudo ${ }^{(10-11)}$. As perguntas norteadoras discorreram sobre as ações realizadas pela equipe de enfermagem, a importância da correta administração de medicamentos e a ocorrência de eventos adversos na unidade. As entrevistas foram gravadas e, posteriormente, transcritas na íntegra.

O registro das informações oriundas das observações foi realizado em um diário de campo, com o intuito de analisar quais ações eram realizadas pela enfermagem para a prática de administração de medicamentos. A carga horária total da observação não participante foi de 40 horas, e foi realizada por uma pesquisadora. A inserção no campo de investigação se deu durante o período de agendamento e realização das entrevistas. A pesquisadora permanecia no campo antes e após a realização das entrevistas, registrando aspectos referentes às ações realizadas durante o processo de administração de medicamentos, o comportamento dos sujeitos da organização e dinâmica do trabalho no setor.

\section{Análise dos dados}

Após a organização das respostas iniciou-se a análise dos dados, que se deu por meio da técnica de Análise de Conteúdo, que tem como objetivo a categorização dos dados para melhor interpretação dos achados no estudo. A análise de conteúdo, segundo Bardin, pode ser compreendida nas seguintes etapas: pré-análise, exploração do material e tratamento dos resultados, inferência e interpretação ${ }^{(12)}$. Os dados registrados no diário de campo foram analisados pela mesma técnica e comparados com o conteúdo transcrito das entrevistas.

Para fins de preservação do anonimato, os técnicos de enfermagem e enfermeiros foram referidos pelas letras TE e E, respectivamente, procedidos do número correspondente à ordem em que foram realizadas as entrevistas (TEl-TE12) e (El-E4).

\section{Procedimentos éticos}

O estudo foi conduzido segundo as normas da Resolução 466/2012 do Conselho Nacional de Saúde, tendo sido aprovado pelo Comitê de Ética em Pesquisa com Seres Humanos da universidade pública federal proponente da pesquisa, sob parecer $n^{\circ}:$ 1.821.022.

As entrevistas ocorreram em ambiente reservado, garantindo a privacidade dos participantes. Antes do início de cada entrevista, foi apresentado e lido para cada entrevistado o Termo de Consentimento Livre e Esclarecido, assinado em duas vias pelos participantes.

\section{RESULTADOS}

A pesquisa foi realizada com 17 profissionais, entre técnicos de enfermagem e enfermeiros, com faixa etária variando entre 23 a 53 anos com média de 49,5 anos. A maioria do sexo feminino (1l participantes). O tempo de serviço na instituição variou entre um mês a nove anos e dois meses, e o período de formação entre um ano a 16 anos com média de oito anos e seis meses.

A partir da análise dos dados, emergiram as seguintes categorias: "Segurança na administração de medicamentos na emergência: ações realizadas" e "Administração segura de medicamentos: desafios vivenciados pela equipe de enfermagem".

\section{Segurança na administração de medicamentos na emer- gência: ações realizadas}

Esta categoria aponta as ações relatadas pela equipe de enfermagem para administração de medicamentos. Os entrevistados referiram seguir as seguintes etapas para uma prática segura:

Pego o nome do paciente direito, carimbo a ETE [sinônimo utilizado para prescrição médica] dele. Coloco meu nome, assino e o horário que estou indo administrar, que é para o outro amigo não administrar não prepará-lo de novo. (TEl)

Olho qual a medicação, a dosagem, qual paciente, em qual via é. Tudo basicamente o que fiz é isso: identifica o paciente, a medicação, a via e a dose. (TE5)

Confirmação do nome do paciente, confirmação da via e da medicação. (E4) 
Identificou-se nas entrevistas que apenas dois enfermeiros citaram os "9 certos" da administração segura de medicamentos, porém não houve detalhamento dos passos a serem seguidos, deixando-os incompletos, conforme demonstrado abaixo:

O que acontece, o paciente ele é abordado primeiramente pelo técnico acolhedor. Na recepção ele faz a ficha. Lá é aferido os sinais vitais, passa pela triagem, passa pelo médico...depois essa ficha vai para a enfermagem. Ai a gente prepara a medicação. Quando vai ser administrado, a dosagem certa... a gente tem lá os 9 certos né? Quando a gente prepara a medicação, confere o nome do paciente, qual medicação, chama o paciente pelo nome, a gente vai arrumar um local para acomodar esse paciente e administrar essa medicação. (E3)

Eu procuro ter certeza do paciente e seguir os 9 certos. Eu, às vezes não consigo fazer todos. Sempre quando está tranquilo eu vou seguir corretamente. Paciente certo, horário certo, via certa, medicação certa e por ai vai..

Durante a observação no campo, foi possível constatar as ações realizadas rotineiramente pela equipe de enfermagem no serviço de emergência, e um fato que merece destaque e que denota práticas inseguras de administração de medicamentos foi observada na admissão, durante a qual os pacientes que chegavam não tinham suas medicações identificadas, sendo preparadas e dispostas nas bandejas sem nenhuma organização. Em casos de pacientes com mesmo nome e sobrenome, a diferenciação se dava através da data de nascimento e nome da mãe presentes no prontuário. Observou-se também que as prescrições médicas eram manuais, com validade de 24 horas e aprazadas pela equipe de enfermagem, que era responsável por separar as medicações diárias de cada paciente. Ressalta-se que não havia padronização no preparo das medicações, o que pode favorecer a ocorrência de eventos adversos. Os dados registrados no diário de campo corroboraram com o conteúdo das entrevistas.

Outra atividade importante que deve ser realizada pelos profissionais de saúde é a lavagem das mãos, que visa interromper a disseminação de microrganismos diminuindo o risco de infecções. No entanto, durante a observação de campo e nas entrevistas, constatou-se que esta era uma conduta frequentemente negligenciada pela equipe de en- fermagem. Apenas alguns entrevistados relataram essa ação como uma etapa da administração segura de medicamentos:

Lavar as mãos, hum... olhar o paciente, se é o paciente certo, via certa, hora certa é...medicação certa, hm...plantão certo (risos). (E2)

Ah! Eu lavo as mãos né? Eu...depois que eu lavo as mãos eu olho a medicação. (TE7)

Então, é... eu vou nos quartos pra ver os pacientes, me apresento pra eles, ai venho na prescrição, lavo as mãos antes de pegar o plantão, venho na prescrição e vejo o que tem pra fazer, se tem alguma prescrição pra aprazar, eu faço né? Aprazo a prescrição, se já está tudo aprazado eu separo tudo que for do meu plantão. (TE12)

\section{Administração segura de medicamentos: desafios viven- ciados pela equipe de enfermagem}

Esta categoria apresenta os desafios vivenciadas pelos profissionais de enfermagem da emergência que possibilitam a ocorrência de erros. $O$ trecho que segue demonstra o exposto:

O que possa vir a me impedir, como eu falei algumas questões atrás é a quantidade de pacientes mesmo. A demanda, a grande demanda prejudica um pouco a sequência correta para a administração de medicamentos. (E5)

Não se observou dificuldades técnicas no preparo dos medicamentos, porém o serviço de emergência se caracterizou por possuir alta demanda de pacientes, ou seja, a dinamicidade do serviço de emergência associada ao alto fluxo de pacientes e a carga de trabalho no setor são situações vivenciadas pela equipe de enfermagem e atuam como barreiras no processo de administração segura de medicamentos. Ressalta-se que por ser um processo que abrange diversas etapas, os erros de medicação podem ser frequentes e consequentemente ocasionar eventos adversos:

Era um medicamento que era para ser administrado na sonda. O comprimido tinha que ser dissolvido e foi feito no acesso central. (TE8)

Embora a maioria dos profissionais entrevistados afirmasse não haver dificuldades no processo de administrar 
medicações, alguns participantes relataram que novos fármacos ou aqueles que não são da rotina do setor atuam como aspectos dificultadores à assistência segura. Tal questão pode ser exemplificada a partir das falas a seguir:

Até hoje não! A não ser que apareça alguma medicação nova, que a gente não conheça né, mas ai a gente é orientado pelo médico pra fazer isso, mas, no mais assim, não tenho dificuldade. (TE2)

Se for uma medicação que eu não conheça [...] a gente vai aprendendo na rotina do ambulatório. Vai conhecendo, mas sempre aparece novidade. (E2)

\section{DISCUSSÃo}

Os resultados encontrados na primeira categoria demonstraram que as ações relatadas pelos participantes do estudo eram realizadas parcialmente quando comparadas ao protocolo do PNSP sobre segurança na prescrição, uso e administração de medicamentos, tendo a maioria citado ações que contemplam os 9 certos da administração, mas de forma incompleta. Sabe-se que a administração é a última barreira possivel para que se possam evitar erros de medicações e danos à saúde do paciente, sendo de extrema importância uniformizar todas as práticas profissionais que envolve o processo de administração de medicamentos ${ }^{(6)}$.

Neste contexto, ressalta-se a importância de padronizar a realização de todas as etapas conhecidas como os "9 certos da administração", sendo necessária a adoção de protocolos específicos para a prevenção de erros. Deve-se destacar o grupo de medicamentos chamados de potencialmente perigosos ou de alta vigilância (high alert medications) que possuem maior potencial de provocar danos no paciente quando existe erro na sua utilização. Destaca-se que na instituição pesquisada não havia nenhum protocolo implementado relacionado à administração de medicamentos ${ }^{(6)}$

Uma pesquisa realizada no Brasil, aponta a elaboração de protocolos e manuais como uma estratégia para minimizar os erros de medicação em unidades de emergência. Nessa lógica, os protocolos para armazenamento, prescrição, dispensação, preparo e administração de medicamentos devem ser implementados, com o objetivo de diminuir a variabilidade na prática clínica e reduzir a incidência dos erros. Assim, torna-se essencial à cultura de segurança a identificação dos motivos pelos quais os protocolos não estão sendo implementados e/ou desenvolvidos adequadamente, para que equipe de enfermagem possa trabalhar de forma sistematizada(4)
Considerando-se o propósito de administrar corretamente as medicações, outras práticas corroboram para que os erros sejam minimizados. A organização das bandejas é uma delas. É necessário que haja a identificação de cada medicação e seus respectivos pacientes, com no mínimo o nome completo e a data de nascimento. Além dessas informações o endereço deve constar na prescrição médica ${ }^{(6)}$. No entanto, durante a observação de campo verificou-se que essas ações não eram realizadas no setor pesquisado.

Perante o protocolo da ANVISA para identificação de pacientes, este deve utilizar a partir de sua admissão hospitalar, uma pulseira de cor branca com seus dados pessoais descritos, devendo o profissional de saúde confirmá-los na iminência da administração medicamentosa ${ }^{(13)}$. Tais práticas vão de encontro aos achados da pesquisa na instituição pesquisada, auxiliando a redução da ocorrência de incidentes.

A tecnologia é uma grande aliada para contribuir efetivamente para a segurança medicamentosa. Programas informatizados podem auxiliar na análise das prescrições medicamentosas, na redução de erro de medicação, tempo de internação e custos hospitalares ${ }^{(7)}$.

A segunda categoria apresentou as dificuldades e desafios que a equipe enfrenta no cotidiano para administração segura dos medicamentos. Pesquisas nacionais e internacionais, citam a escassez de profissionais e a sobrecarga de trabalho da equipe de enfermagem como fatores que dificultam a administração segura de medicamentos ${ }^{(14-15)}$, e estes dados podem ser comparados à realidade desta pesquisa enfatizada pelas palavras "quantidade", "demanda" e "prejudica" contidas nas falas. Os serviços de emergência destacam-se por possuírem tais características, pelo fato de atuar como alternativa de atendimento à população em diversas situações, fazendo com que o setor se torne suscetivel à ocorrência de eventos adversos ${ }^{(15)}$.

No sul do Brasil foi realizado um estudo objetivando caracterizar os incidentes medicamentosos no serviço de emergência. A administração de medicamentos foi o processo relacionado à maioria dos incidentes ocorridos devido ao elevado nível de raciocínio, concentração e memória requerida nessa atividade. Além de mudanças estruturais, o dimensionamento de pessoal de enfermagem pode minimizar distrações contribuindo para a redução de incidentes $^{(16)}$

Pesquisa aponta que os fatores de risco para o erro de medicação podem ser relacionados à ausência de informações sobre o medicamento, dosagem, diluição e aprazamento. Esses dados corroboram com os achados dessa 
pesquisa, pois alguns participantes relataram a dificuldade em administrar medicamentos que não eram do seu cotidiano assistencial, pois desconheciam informações essenciais para a administração segura ${ }^{(17)}$.

Para reduzir o erro é necessário que os hospitais busquem estratégias de intervenção, dentre elas destacam-se: estratégias educacionais (realização de campanhas, elaboração de manuais explicativos, criação de comissão multidisciplinar envolvida com a prevenção e redução dos eventos adversos aos medicamentos); estratégias organizacionais (reuniões, criação de protocolos e mudanças no processo de trabalho) e novas tecnologias (implementação de prescrição por sistema informatizado, implantação da dose unitária e do código de barras na administração de medicamentos). Assim, torna-se essencial a discussão de atividades de educação em saúde nos ambientes de trabalho(4)

Diante das questões supracitadas e de outros problemas referentes à segurança do paciente, o MS, através da Portaria n.1996/2007, dispôs sobre as diretrizes para implementação da Política Nacional de Educação Permanente em Saúde. Uma das ações previstas por esta política é a consolidação da educação permanente (EP) nos serviços de saúde, partindo das necessidades encontradas pela equipe de saúde e usuários, visando a resolutividade e integralidade de ações por meio de estratégias/metodologias de trabalho participativas, dialógicas e contínuas ${ }^{(18)}$.

Há que se ressaltar que a EP constitui uma importante estratégia de formação coletiva, ao se constituir como espaço de revisão de conceitos, reflexões sobre a prática, diálogo interdisciplinar, sendo um potente recurso de formação em serviço(19). Ademais, almeja, por meio da problematização da realidade, mudanças nas práticas dos trabalhadores, discutidas e pactuadas no grupo de EP, por meio da definição de competências mínimas a serem desenvolvidas. É relevante destacar que o compartilhamento de situações/desafios vivenciados no cotidiano dos serviços e a busca de caminhos para sua resolução dependem de um efetivo trabalho em equipe, transdisciplinar, que compartithe as responsabilidades e se corresponsabilize pela reorganização dos processos de trabalho(19-20).

Dessa maneira, além de processos que permitam incorporar tecnologias e referenciais necessários, é preciso implementar espaços de discussão, análise e reflexão da prática no cotidiano de trabalho e dos referenciais que orientam essas práticas, considerando que o cotidiano é um espaço sempre aberto à revisão permanente $\mathrm{e}^{(21)}$

O enfermeiro enquanto responsável por sua equipe tem um papel fundamental para garantia de uma assistência livre de danos, e deve ter a iniciativa de promover a EP para melho- rar a assistência e prevenir a incidência de erros. Cabe à instituição incentivar a EP, permitindo uma reflexão crítica sobre a atuação ética frente aos erros de medicação, oferecendo recursos para tal, melhorando a qualidade técnico-científico dos profissionais e garantindo a qualidade na assistência prestada na instituição e a segurança do paciente ${ }^{(22)}$

\section{Limitações da pesquisa}

A presente pesquisa apresenta como limitação ter sido realizada em uma única instituição com cultura específica não sendo cabível generalização.

\section{Contribuições para a prática}

Esta pesquisa contribui para a temática ao demonstrar as dificuldades vivenciadas na administração segura de medicamentos na emergência o que pode resultar em eventos adversos nesse cenário de atenção à saúde. Almeja-se que este estudo proporcione elementos relevantes para subsidiar novos estudos e novas discussões voltadas para a prática segura na assistência à saúde.

\section{CONSIDERAÇÕES FINAIS}

Os achados da presente pesquisa sinalizam que a equipe de enfermagem entrevistada atende parcialmente às normas de administração segura dos medicamentos, visto que atua em convergência com algumas ações recomendadas pelo PNSP; no entanto, a falta de padronização para a administração dos medicamentos, o alto fluxo de pacientes, a sobrecarga de trabalho e o surgimento de novos medicamentos podem favorecer a ocorrência de eventos adversos.

Ademais, apesar do fomento para implantação da cultura de segurança do paciente nos serviços de saúde, a instituição pesquisada não apresentava nenhum protocolo sobre a administração de medicamentos, o que pode trazer prejuízos às práticas seguras, pela ausência de padronização para o dispensação, preparo e administração das medicações, favorecendo a ocorrência de eventos adversos.

Novas estratégias precisam ser implementas no setor como o investimento em novas tecnologias e a implementação da educação permanente, a fim de capacitar a equipe acerca da administração segura de medicamentos, e seu formato deve considerar questões trazidas da prática, de forma a buscar reflexões e possiveis alternativas para a complexidade dos problemas que emergem do cotidiano dos serviços.

\section{Contribuições dos autores:}

Todos os autores contribuíram para o manuscrito. 


\section{REFERÊNCIAS}

1- World Health Organization. World Alliance for Patient Safety. Global Patient Safety Challenge: 2005-2006 [Internet]. Genova: WHO; 2005 [cited 2017 Aug 11]. Available from: https://apps.who.int/iris/bitstream/handle/10665/43358/9241593733_eng.pdf?sequence=1EisAllowed=y

2- Ministério da Saúde (BR). Gabinete do Ministro. Portaria 529, de 1 de abril de 2013. Institui o Programa Nacional de Segurança do Paciente (PNSP) [Internet]. Diário Oficial da União: República Federativa do Brasil; 2013. Mar 04. [cited 2018 Jan] Seção 1: 43-44 p. (col. 3). Available from: https://bvsms. saude.gov.br/bvs/saudelegis/gm/2013/prt0529_01_04_2013.html

3- Agência Nacional de Vigilância Sanitária. RDC n³6 de 25 de julho de 2013. Institui ações para segurança do paciente em serviços de saúde e dá outras providências. ANVISA [Internet], Brasilia (DF), 26 jul 2013 [cited 2017 Apr 4]. Available from: https://bvsms.saude.gov.br/bvs/saudelegis/anvisa/2013/rdc0036_25_07_2013.html

4- Mieiro DB, Oliveira EBC, Fonseca REP, Mininel VA, Zem-Mascarenhas SH, Machado RC. Estratégias para minimizar erros de medicação em unidades de emergência: revisão integrativa. Rev. Bras. Enferm. [Internet]. 2019 Fev [cited 2019 Dec 03];72 (Suppl 1): 307-314. Available from: https://www.scielo.br/scielo.php?script=sci_arttextEpid=\$0034-71672019000700307\&lng=pt.\%20\%20http://dx.doi.org/10.1590/0034-7167-2017-0658

5- Agência Nacional de Vigilância Sanitária. Pacientes pela segurança do paciente em serviços de saúde: Como posso contribuir para aumentar a segurança do paciente? Orientações aos pacientes, familiares e acompanhantes/ ANVISA. Brasilia [Internet]. 2017 [cited 2018 Jan 05]. Available from: http://portal.anvisa.gov.br/documents/33852/3507912/Como+posso+contribuir+para+aumentar+a+segurança+do+paciente/52efbd76-b692-4b0e-8b 70-6567e532a716

6- Ministério da Saúde (BR). Protocolo de segurança na prescrição, uso e administração de medicamentos; Ministério da Saúde [Internet]. 2013 [cited 2018 Jan 06]. Available form: https://www20.anvisa.gov.br/segurancadopaciente/index.php/publicacoes/item/seguranca-na-prescricao-uso-e-administracao-de-medicamentos

7- Etelvino MAL, Santos ND, Aguiar BGC, Assis TG. Segurança do paciente: uma análise do aprazamento de medicamentos. Rev. Enfermagem em foco [intetnet]. 2019 [cited 2020 May 6];10 (4): 87-92. Available from: http://revista. cofen.gov.br/index.php/enfermagem/article/view/2251

8- Santos JLG, Lima MADS, Pestana AL, Colomé ICS, Erdmann AL. Estratégias utilizadas pelos enfermeiros para promover o trabalho em equipe em um serviço de emergência. Rev. Gaúcha Enferm. [Internet]. 2016 fev: [cited 2017 May 6];37(1). Available froml: https://www.scielo.br/pdf/rgenf/ v37nl/0102-6933-rgenf-1983-144720160150178.pdf

9- Azevedo KCC, Alves AMPM, Félix ZC. Implantação do Núcleo de Segurança do Paciente em um serviço de saúde. Rev enferm UFPE [Internet]. 2016 [cited 2017 May 19]; 10(12). Available from: https://periodicos.ufpe.br/ revistas/revistaenfermagem/article/viewFile/11540/13448

10- Minayo MCS. O desafio do conhecimento: pesquisa qualitativa em saúde. 14⿳亠口冋. ed. São Paulo: Hucitec; 2014.

11- Depresbiteris L, Tavares MR. Diversificar é preciso: instrumentos e téc- nicas de avaliação de aprendizagem. São Paulo: Editora Senac São Paulo: 2009

12- Bardin L. Análise de conteúdo. Lisboa: Edições 70; 2011.

13- Ministério da Saúde (BR). Protocolo de identificação do paciente; Ministério da Saúde/ANVISA/Fio Cruz [internet]. 2013 [cited 2017 Aug 13]. Available from: https://www20.anvisa.gov.br/segurancadopaciente/index.php/ publicacoes/item/identificacao-do-paciente

14- Lawton R, Carruthers S, Gardner P. Identifying the Latent Failures Underpinning Medication Administration Errors: An Exploratory Study. Health Serv Res. [Internet] 2012 fev; [cited 2017 set 13]; 47(4). Disponivel: http:// onlinelibrary.wiley.com/doi/10.1111/j.1475-6773.2012.01390.x/abstract;jsessionid=FlFAB5E91678189478F2C777442FC510.f04t04

15- Pereira CDFD, Tourinho FSV, Santos VEP. Segurança do paciente: avaliação do sistema de medicação por enfermeiros utilizando análise fotográfica. Enferm. Foco [internet]. 2016 [cited 2018 mar 19]; 7(1): 76-80. Available from: http://revista.cofen.gov.br/index.php/enferma\%20gem/article/view/672

16- Valle MMF, Cruz EDA, Santos T. Incidentes com medicamentos em unidade de urgência e emergência: análise documental. Rev. esc. enferm. USP [Internet]. 2017 [cited 2018 Jun 18]; 51: e03271. Disponivel: http://www.scielo. br/scielo.php?script=sci_arttext\&pid=S0080-623420170001004698Ing=en

17- Souza AFR, Queiroz JC, Vieira AN, Solon LGS, Bezzerra ELSF. Os erros de medicação e os fatores de risco associados à sua prescrição. Rev. Enferm. Foco [internet]. 2019 [cited 2020 May 06]; 10 (4): 12-16. Available from: http://revista.cofen.gov.br/index.php/enfermagem/article/view/1900

18- Ministério da Saúde (BR). Portaria GM/MS n¹.996 de 20 de agosto de 2007. Dispõe sobre as diretrizes para a implementação da Política Nacional de Educação Permanente em Saúde. Diário Oficial da União [periódico internet], Brasilia (DF). 20 ago 2007 [cited 2017 May 5] Available from: http://bvsms.saude.gov.br/bvs/saudelegis/gm/2007/ prt1996_20_08_2007.html

19- Silva DLS, Knobloch FA. A equipe enquanto lugar de formação: a educação permanente em um Centro de Atenção Psicossocial Álcool e outras drogas. Interface (Botucatu) [internet]. 2016 Apr./June [cited 2018 Jul 12]; 20(57): 325-335. Available from: https://www.scielo.br/scielo.php?script=sci_arttext\&pid=S1414-32832016000200325

20- França T, Medeiros KR, Belisario AS, Garcia AC, Pinto ICM, Castro JL, Pierantoni CR. Política de Educação Permanente em Saúde no Brasil: a contribuição das Comissões Permanentes de Integração Ensino-Serviço. Ciênc. saúde coletiva [Internet]. 2017 [cited 2017 agu 08]: 22(6). Available from: https://www.scielo.br/pdf/csc/v22n6/1413-8123-csc-22-06-1817.pdf

21- Ceccim RB. Educação Permanente em Saúde: desafio ambicioso e necessário. Interface - Comunicação, Saúde, Educação [Internet] 2005 set/ fev; [cited 2017 out 20]; 9(16). Available from: https://lume.ufrgs.br/handle/10183/129275

22- Mangilli DC, Assunção MT, Zanini MTB, Dagostin VS, Saratto MT. Atuação ética do enfermeiro frente aos erros de medicação. Enferm. Foco [Internet]. 2017; [cited 2018 fev 20]; 8(1): 62-66. Available from: http://revista.cofen. gov.br/index.php/enfermagem/article/view/878 